\title{
Bioefficacy of Synthetic Insecticides, Bio-Pesticide and Botanical against Thrips and Watermelon Bud Necrosis Viral Disease on Watermelon
}

\author{
C.R. Shruthi*, G.B. Narabenchi, B.S. Rohini and Chethan Nandi \\ University of Horticultural Sciences, Bagalkot, Udyangiri, Bagalkot-587 102, India \\ *Corresponding author
}

A B S T R A C T

\begin{abstract}
Keywords
Thrips palmi,

Watermelon

budnecrosis virus,

Bio-efficacy.

Article Info

Accepted:

04 July 2017

Available Online:

10 September 2017

A field experiment conducted during summer season of 2014 revealed that, as the crop stage advanced the extent of thrips infestation and WBNV disease incidence also increased irrespective of the treatments. Thereafter, the thrips population declined gradually. The overall disease incidence in the plots assigned to different treatments did not exceed 45 per cent including control plots at initial stage of the crop growth i.e. up to 60 days after sowing. Thereafter, the disease incidence increased significantly and reached about 100 per cent in all the treatment plots within a short period of time.
\end{abstract}

\section{Introduction}

Watermelon, Citrullus lanatus (Thunb.) is a well-known vegetable of summer season, indigenous to tropical Africa. World over watermelon is grown in an area of 3.69 million hectares with an estimated annual production of 97.43 million tonnes and average productivity of 26.37 tonnes per hectare.

Leading watermelon growing countries in the world are China, Turkey, Iran, Brazil, USA, Egypt, Russian federation and Mexico. In India, watermelon is being grown since prehistoric period. It is a major crop of various river beds in Uttar Pradesh, Punjab, Haryana, Rajasthan, Bihar, Gujarat, Maharashtra, Andhra Pradesh and Karnataka. In India, it occupies an area of about 20,000 hectares with an annual production of 2.55 lakh tonnes and productivity of 12.75 tonnes per hectare (http://www.faostat.fao.org).

Watermelon is a common man's fruit relished by both rich and poor alike.

The fruit contains 95 per cent water, 0.2 per cent protein, 0.3 per cent minerals and 3.3 per cent carbohydrates per $100 \mathrm{~g}$ fresh weight (Aykroyd, 1963). Watermelon is rich in iron content among all the cucurbitaceous crops.

Most of the cultivars have deep pink or pale pink flesh colour with slightly reddish tinge containing largely lycopene and anthocyanin pigments. It is popular especially in hot weather, for its sweet and juiciness. 
Watermelon thrips, Thripspalmi Karny is one of the most important pests as it is posing the problems both as a pest and vector for transmitting a number of tospoviruses and in turn causing bud necrosis viral diseases in many agricultural and horticultural crops (Mound, 1996). Watermelon Bud Necrosis Viral disease is one such dreaded viral disease regularly appearing in major watermelon growing areas of Karnataka. The occurrence of bud necrosis disease on watermelon was observed for the first time in India by Singh and Krishnareddy (1995) during the growing season of 1991-92. Since then, bud necrosis disease has been observed in different watermelon growing areas of Karnataka and is on increasing trend year after year. Now the disease has attained a major status and has become an endemic causing enormous loss in yield and quality of watermelon. Hence, the present study was undertaken to evaluate synthetic insecticides, bio-pesticide and botanical against thrips and WBNV disease on watermelon crop.

\section{Materials and Methods}

The experiment was laid out in Randomised Block Design consisting of twelve treatments replicated thrice. Each plot size was about 12 m X $3 \mathrm{~m}$. A total of five sprays were given starting from 45 days after sowing of seeds by using power operated Knapsack sprayer at weekly interval. The treatment details are as follows (Table 1).

The seeds were treated with a solution of imidacloprid 45 FS @ $10 \mathrm{ml}$ mixed in $25 \mathrm{ml}$ of water per $\mathrm{kg}$ of seeds and the treated seeds were shade dried. Then these treated seeds were sown in assigned plots. Pre-treatment count was made prior to the spray. The post treatment counts were made at one, three and seven days after each spray. Observations were made on number of thrips and the WBNV disease incidence. For recording thrips infestation, five plants were selected randomly in each replication and counted the population by tapping the growing tips on a stiff black paper board $(30 \times 30 \mathrm{~cm})$. The extent of WBNV disease incidence was recorded based on symptoms exhibited on plants like leaf mottling, yellowing and necrotic spots on leaves, necrotic streaks on vein, petiole and stem, shortened internode, necrosis of the terminal bud. A total number of plants were recorded and among these plants, number of plants infected with WBNV was also recorded. Then, the per cent disease incidence was calculated by using following formula.

No. of plants infected

Per cent disease incidence $=$------------- x 100

Total no. of plants observed

Data was analyzed by using one way ANOVA and two factor ANOVA. The treatment means were separated by using DMRT.

\section{Results and Discussion}

A field experiment conducted during summer season of 2014 revealed that, as the crop stage advanced the extent of thrips infestation and WBNV disease incidence also increased irrespective of the treatments. Thereafter, the thrips population declined gradually. The overall disease incidence in the plots assigned to different treatments did not exceed 45 per cent including control plots at initial stage of the crop growth i.e. up to 60 days after sowing. Thereafter, the disease incidence increased significantly and reached about 100 per cent in all the treatment plots within a short period of time.

Among the different synthetic insecticides, biopesticide and botanical pesticide evaluated, the imidacloprid @ $10 \mathrm{ml}$ per kg seeds as seed treatment followed by foliar spraying of 
acephate @ $1 \mathrm{~g} / \mathrm{l}$ of water found to be superior in reducing the thrips population throughout the cropping period. Next best treatments in the order were diafenthiuron $1.25 \mathrm{~g} / \mathrm{l}$ of water and imidacloprid $0.30 \mathrm{ml} / \mathrm{l}$ of water as foliar sprays (Table 2).

Interestingly, though the dimethoate is relatively older insecticide molecule, in the present investigation it was found as effective as other new molecules of insecticides like abamectin, imidacloprid and thiamethoxam in reducing the thrips population at seven days after first spray (Table 2).

Overall, in second spray the silver colour UV reflective mulch, imidacloprid @ $10 \mathrm{ml}$ per $\mathrm{kg}$ seeds as seed treatment followed by acephate @ $1.00 \mathrm{~g} / \mathrm{l}$ of water as foliar spray were found to be effective in reducing thrips infestation on watermelon (Table 2). Similar trend was noticed in seventh day after third spray.

Overall in fourth spray, imidacloprid @ $10 \mathrm{ml}$ per $\mathrm{kg}$ seeds as seed treatment followed by foliar spray of acephate @ $1.00 \mathrm{~g} / \mathrm{l}$ of water found to be effective in managing thrips and a fungal bio-pesticide, Lecanicillium lecanii recorded the maximum thrips infestation (Table 2).

Overall in fifth spray, the lowest thrips infestation was recorded in imidacloprid @ 10 $\mathrm{ml}$ per $\mathrm{kg}$ seeds as seed treatment followed by foliar spray of acephate @ 1.00 g/l of water. As observed in earlier sprays, again a maximum thrips infestation $(25.00$ mean number of thrips/5 plants) was recorded with a bio-pesticide, Lecanicillium lecanii @ 2.00 $\mathrm{g} / \mathrm{l}$ of water (Table 2).

Similar trend was noticed with respect to the performance of various treatments on thrips when the data were analysed using two factorial analysis. Among the different treatments tested, the seed treatment with imidacloprid followed by foliar application of acephate found to be effective in reducing the thrips populations on watermelon.

In the case of different sprays the lowest pooled mean number of thrips 2.68 per plant was recorded in first spray, whereas highest pooled mean number of thrips 25.63 per plant was recorded with third spray (Table 3 ).

Table.1 Details of treatments imposed against thrips and WBNV disease incidence in watermelon crop

\begin{tabular}{|l|l|l|}
\hline Treatments & Particulars & Dosage (ml or g/l of water) \\
\hline T1 & Azadirachtin 1500 ppm & $3.0 \mathrm{ml}$ \\
\hline T2 & Lecanicillium (=Verticilium) lechanii & $2.0 \mathrm{~g}$ \\
\hline T3 & $\begin{array}{l}\text { Imidacloprid 45 FS (Seed Treatment) } \\
\text { Acephate 75 SP (Foliar spray) }\end{array}$ & $\begin{array}{l}10 \mathrm{ml}+25 \mathrm{ml} \text { of water/Kg } \\
\text { Seeds }+1.0 \mathrm{~g} / 1\end{array}$ \\
\hline T4 & Diafenthiuron 50 WP & $1.25 \mathrm{~g}$ \\
\hline T5 & Fipronil 5 SC & $1.0 \mathrm{ml}$ \\
\hline T6 & Abamectin 1.9 SC & $0.25 \mathrm{ml}$ \\
\hline T7 & Imidacloprid 30.50 SC & $0.30 \mathrm{ml}$ \\
\hline T8 & Thiomethoxam 25 WG & $0.20 \mathrm{~g}$ \\
\hline T9 & Dimethoate 30 EC & $1.70 \mathrm{ml}$ \\
\hline T10 & Lambda cyahalothrin 5 EC & $0.50 \mathrm{ml}$ \\
\hline T11 & Acephate 75 SP & $1.0 \mathrm{~g}$ \\
\hline T12 & Untreated control (Water Spray) & - \\
\hline
\end{tabular}


Table.2 Bio-efficacy of different chemical insecticides, bio-pesticide and botanical pesticide against Thrips palmi on watermelon crop

\begin{tabular}{|c|c|c|c|c|c|c|c|c|c|c|c|c|c|c|c|c|c|}
\hline \multirow{3}{*}{ Treatment } & \multirow{3}{*}{$\begin{array}{c}\text { Dosage } \\
\text { ml or } \\
\text { g/lt }\end{array}$} & \multicolumn{16}{|c|}{ *Mean no. of thrips/5 plants } \\
\hline & & \multicolumn{4}{|c|}{ I Spray } & \multicolumn{3}{|c|}{ II Spray } & \multicolumn{3}{|c|}{ III Spray } & \multicolumn{3}{|c|}{ IV Spray } & \multicolumn{3}{|c|}{ V Spray } \\
\hline & & $\begin{array}{l}\text { Before } \\
\text { spray }\end{array}$ & 1 DAS & 3DAS & 7 DAS & 1 DAS & 3 DAS & 7 DAS & 1 DAS & 3 DAS & 7 DAS & 1 DAS & 3 DAS & 7 DAS & 1DAS & 3 DAS & 7 DAS \\
\hline $\mathrm{T}_{1}$ & $3 \mathrm{ml}$ & $\begin{array}{c}9.67 \\
(2.94) \\
\end{array}$ & $\begin{array}{c}3.67 \\
(1.97)^{\mathrm{b}} \\
\end{array}$ & $\begin{array}{c}1.00 \\
(1.09)^{\mathrm{bc}}\end{array}$ & $\begin{array}{r}8.00 \\
(2.66)^{\mathrm{b}} \\
\end{array}$ & $\begin{array}{c}8.67 \\
(3.00)^{\text {ab }} \\
\end{array}$ & $\begin{array}{c}27.33 \\
(4.83)^{\text {ab }} \\
\end{array}$ & $\begin{array}{r}49.67 \\
(6.76){ }^{\text {ab }} \\
\end{array}$ & $\begin{array}{c}15.67 \\
(3.69){ }^{\mathrm{bc}} \\
\end{array}$ & $\begin{array}{c}39.33 \\
(5.87)^{\mathrm{ab}} \\
\end{array}$ & $\begin{array}{c}34.33 \\
(5.79){ }^{\text {bcd }} \\
\end{array}$ & $\begin{array}{c}22.00 \\
(4.67){ }^{\mathrm{abc}} \\
\end{array}$ & $\begin{array}{c}30.33 \\
(5.51)^{\mathrm{b}} \\
\end{array}$ & $\begin{array}{c}23.67 \\
(5.79)^{\text {bcd }} \\
\end{array}$ & $\begin{array}{r}22.67 \\
(4.76){ }^{\text {bc }} \\
\end{array}$ & $\begin{array}{c}16.00 \\
(3.99)^{\text {bcd }}\end{array}$ & $\begin{array}{c}22.00 \\
(4.67)^{\mathrm{bc}} \\
\end{array}$ \\
\hline $\mathrm{T}_{2}$ & $2 \mathrm{~g}$ & $\begin{array}{c}5.67 \\
(2.47) \\
\end{array}$ & $\begin{array}{c}2.67 \\
(1.64)^{\mathrm{bc}} \\
\end{array}$ & $\begin{array}{c}0.67 \\
(0.99)^{\mathrm{bc}} \\
\end{array}$ & $\begin{array}{c}5.67 \\
(2.47) \mathrm{bc} \\
\end{array}$ & $\begin{array}{c}8.33 \\
(2.66)^{\mathrm{bc}} \\
\end{array}$ & $\begin{array}{c}18.00 \\
(4.22)^{\mathrm{abc}} \\
\end{array}$ & $\begin{array}{c}33.33 \\
(5.68)^{\mathrm{bc}} \\
\end{array}$ & $\begin{array}{c}25.00 \\
(4.93)^{\mathrm{ab}} \\
\end{array}$ & $\begin{array}{c}38.00 \\
(5.79)^{a b} \\
\end{array}$ & $\begin{array}{c}52.67 \\
(7.25)^{\text {ab }} \\
\end{array}$ & $\begin{array}{c}28.00 \\
(5.27)^{\mathrm{ab}} \\
\end{array}$ & $\begin{array}{c}28.33 \\
(5.32)^{\mathrm{bc}} \\
\end{array}$ & \begin{tabular}{|c|}
31.00 \\
$(7.25)^{\mathrm{ab}}$ \\
\end{tabular} & $\begin{array}{c}26.67 \\
(5.14)^{\text {ab }} \\
\end{array}$ & $\begin{array}{c}20.33 \\
(4.51)^{\mathrm{b}} \\
\end{array}$ & $\begin{array}{c}25.00 \\
(4.93)^{\mathrm{b}} \\
\end{array}$ \\
\hline $\mathrm{T}_{3}$ & $\begin{array}{c}10 \mathrm{ml}+ \\
1 \mathrm{~g}\end{array}$ & $\begin{array}{c}5.67 \\
(2.47) \\
\end{array}$ & $\begin{array}{c}0.33 \\
(0.88)^{\mathrm{d}} \\
\end{array}$ & $\begin{array}{c}0.00 \\
(0.70)^{\mathrm{c}} \\
\end{array}$ & $\begin{array}{c}1.00 \\
(1.09)^{\mathrm{cd}} \\
\end{array}$ & $\begin{array}{c}0.33 \\
(0.88)^{\mathrm{e}} \\
\end{array}$ & $\begin{array}{c}2.33 \\
(1.5)^{\mathrm{d}} \\
\end{array}$ & $\begin{array}{c}9.67 \\
(2.75)^{\mathrm{e}} \\
\end{array}$ & $\begin{array}{c}2.00 \\
(1.41)^{\mathrm{d}} \\
\end{array}$ & $\begin{array}{c}13.00 \\
(3.11)^{\text {def }}\end{array}$ & $\begin{array}{c}20.00 \\
(4.41)^{\mathrm{d}} \\
\end{array}$ & $\begin{array}{c}8.67 \\
(2.90)^{\mathrm{d}} \\
\end{array}$ & $\begin{array}{c}9.00 \\
(2.97)^{\mathrm{g}} \\
\end{array}$ & $\begin{array}{c}8.33 \\
(4.41)^{\mathrm{d}} \\
\end{array}$ & $\begin{array}{c}5.67 \\
(2.36)^{\mathrm{f}} \\
\end{array}$ & $\begin{array}{c}5.00 \\
(2.23)^{\mathrm{f}} \\
\end{array}$ & $\begin{array}{r}9.00 \\
(3.00)^{\mathrm{e}} \\
\end{array}$ \\
\hline $\mathrm{T}_{4}$ & $1.25 \mathrm{~g}$ & $\begin{array}{c}4.33 \\
(2.01) \\
\end{array}$ & $\begin{array}{c}0.33 \\
(0.88)^{\mathrm{d}} \\
\end{array}$ & $\begin{array}{c}0.00 \\
(0.70)^{\mathrm{c}} \\
\end{array}$ & $\begin{array}{c}0.33 \\
(0.88)^{\mathrm{d}} \\
\end{array}$ & $\begin{array}{c}0.33 \\
(0.88)^{\mathrm{e}} \\
\end{array}$ & $\begin{array}{c}6.00 \\
(2.46)^{\mathrm{cd}} \\
\end{array}$ & \begin{tabular}{|c|}
28.33 \\
$(5.07)$ bcd \\
\end{tabular} & $\begin{array}{c}17.33 \\
(3.98)^{\mathrm{bc}} \\
\end{array}$ & $\begin{array}{c}7.33 \\
(2.49)^{\text {ef }} \\
\end{array}$ & $\begin{array}{c}24.33 \\
(4.92)^{\mathrm{cd}} \\
\end{array}$ & $\begin{array}{c}15.00 \\
(3.86)^{\mathrm{cd}} \\
\end{array}$ & $\begin{array}{c}4.00 \\
(3.7)^{\mathrm{f}} \\
\end{array}$ & $\begin{array}{c}20.00 \\
(4.92)^{\mathrm{cd}} \\
\end{array}$ & $\begin{array}{c}14.67 \\
(3.81)^{\mathrm{de}} \\
\end{array}$ & $\begin{array}{c}10.00 \\
(3.11)^{\text {def }} \\
\end{array}$ & $\begin{array}{c}13.67 \\
(3.68)^{\mathrm{de}}\end{array}$ \\
\hline $\mathrm{T}_{5}$ & $1 \mathrm{ml}$ & $\begin{array}{c}6.67 \\
(2.37) \\
\end{array}$ & $\begin{array}{c}1.00 \\
(1.18)^{\mathrm{cd}} \\
\end{array}$ & $\begin{array}{c}1.00 \\
(1.09)^{\mathrm{bc}} \\
\end{array}$ & $\begin{array}{c}0.33 \\
(0.88)^{\mathrm{d}} \\
\end{array}$ & $\begin{array}{c}2.33 \\
(1.65)^{\text {de }} \\
\end{array}$ & $\begin{array}{c}9.67 \\
(2.86)^{\text {bcd }} \\
\end{array}$ & $\begin{array}{c}12.00 \\
(3.36)^{\mathrm{de}} \\
\end{array}$ & $\begin{array}{c}17.00 \\
(3.92)^{\mathrm{bc}} \\
\end{array}$ & $\begin{array}{c}2.33 \\
(1.56)^{\mathrm{f}} \\
\end{array}$ & $\begin{array}{c}30.00 \\
(5.43)^{\mathrm{bcd}}\end{array}$ & $\begin{array}{c}15.33 \\
(3.85)^{\mathrm{cd}} \\
\end{array}$ & $\begin{array}{c}19.00 \\
(4.35)^{\mathrm{e}} \\
\end{array}$ & $\begin{array}{c}16.67 \\
(5.43)^{\text {bcd }} \\
\end{array}$ & $\begin{array}{c}17.67 \\
(4.18)^{\text {cde }} \\
\end{array}$ & $\begin{array}{c}9.00 \\
(2.97){ }^{\text {ef }} \\
\end{array}$ & $\begin{array}{c}17.67 \\
(4.17)^{\text {bcd }} \\
\end{array}$ \\
\hline $\mathrm{T}_{6}$ & $0.25 \mathrm{ml}$ & $\begin{array}{c}5.33 \\
(2.35) \\
\end{array}$ & $\begin{array}{c}0.67 \\
(1.05)^{\mathrm{cd}} \\
\end{array}$ & $\begin{array}{c}0.67 \\
(1.05)^{\mathrm{bc}} \\
\end{array}$ & $\begin{array}{c}3.33 \\
(1.94)^{\mathrm{bcd}} \\
\end{array}$ & $\begin{array}{c}4.00 \\
(1.97)^{\mathrm{cd}}\end{array}$ & \begin{tabular}{|c|}
13.33 \\
$(3.57)^{\mathrm{bcd}}$ \\
\end{tabular} & $\begin{array}{c}25.00 \\
(4.84)^{\mathrm{cd}} \\
\end{array}$ & $\begin{array}{c}15.33 \\
(3.90)^{\mathrm{bc}} \\
\end{array}$ & $\begin{array}{c}13.67 \\
(3.21)^{\text {cdef }} \\
\end{array}$ & \begin{tabular}{|c|}
26.67 \\
$(5.07)^{\mathrm{cd}}$ \\
\end{tabular} & $\begin{array}{c}21.33 \\
(4.59)^{\mathrm{abc}} \\
\end{array}$ & $\begin{array}{c}26.67 \\
(5.14)^{\mathrm{bcd}} \\
\end{array}$ & \begin{tabular}{|c|}
23.00 \\
$(5.08)^{\mathrm{cd}}$ \\
\end{tabular} & $\begin{array}{c}20.33 \\
(4.43)^{\text {bcd }} \\
\end{array}$ & \begin{tabular}{|c|}
16.33 \\
$(4.00)^{\text {bcd }}$ \\
\end{tabular} & $\begin{array}{c}24.00 \\
(4.89)^{\mathrm{b}}\end{array}$ \\
\hline $\mathrm{T}_{7}$ & $0.30 \mathrm{ml}$ & $\begin{array}{r}5.33 \\
(2.14) \\
\end{array}$ & $\begin{array}{c}1.00 \\
(1.23)^{\text {cd }} \\
\end{array}$ & $\begin{array}{c}2.00 \\
(1.43)^{\mathrm{b}} \\
\end{array}$ & $\begin{array}{c}3.33 \\
(1.90)^{\mathrm{bcd}} \\
\end{array}$ & $\begin{array}{c}3.33 \\
(1.89)^{\mathrm{cd}} \\
\end{array}$ & $\begin{array}{c}21.00 \\
(4.41)^{\mathrm{abc}} \\
\end{array}$ & $\begin{array}{c}22.67 \\
(4.42)^{\text {cde }} \\
\end{array}$ & $\begin{array}{r}11.33 \\
(3.29)^{\mathrm{c}} \\
\end{array}$ & $\begin{array}{c}7.00 \\
(2.51)^{\text {ef }} \\
\end{array}$ & $\begin{array}{c}28.33 \\
(5.29)^{\mathrm{cd}} \\
\end{array}$ & $\begin{array}{c}8.33 \\
(2.77)^{\mathrm{d}} \\
\end{array}$ & $\begin{array}{r}9.00 \\
(2.18)^{\mathrm{g}} \\
\end{array}$ & $\begin{array}{c}10.67 \\
(5.29)^{\mathrm{cd}} \\
\end{array}$ & $\begin{array}{c}12.33 \\
(3.48)^{\mathrm{e}} \\
\end{array}$ & $\begin{array}{c}9.33 \\
(3.03)^{\text {ef }} \\
\end{array}$ & $\begin{array}{c}16.00 \\
(4.00)^{\mathrm{cd}}\end{array}$ \\
\hline $\mathrm{T}_{8}$ & $0.20 \mathrm{~g}$ & $\begin{array}{c}6.00 \\
(2.30) \\
\end{array}$ & $\begin{array}{c}0.33 \\
(0.88)^{\mathrm{d}} \\
\end{array}$ & $\begin{array}{c}0.33 \\
(0.88)^{\mathrm{bc}} \\
\end{array}$ & $\begin{array}{c}4.00 \\
(1.85)^{\mathrm{bcd}} \\
\end{array}$ & $\begin{array}{c}2.00 \\
(1.47)^{\mathrm{de}}\end{array}$ & $\begin{array}{c}22.00 \\
(4.33)^{\mathrm{abc}} \\
\end{array}$ & \begin{tabular}{|c|}
31.67 \\
$(5.37))^{\mathrm{bc}}$ \\
\end{tabular} & $\begin{array}{c}13.33 \\
(3.6)^{\mathrm{bc}}\end{array}$ & $\begin{array}{c}34.67 \\
(5.12)^{\mathrm{abc}} \\
\end{array}$ & $\begin{array}{c}46.00 \\
(6.55){ }^{\mathrm{bc}} \\
\end{array}$ & $\begin{array}{c}19.33 \\
(4.32)^{\mathrm{bc}} \\
\end{array}$ & $\begin{array}{c}21.00 \\
(3.73)^{\mathrm{de}}\end{array}$ & $\begin{array}{c}22.67 \\
(6.55)^{\mathrm{bc}} \\
\end{array}$ & $\begin{array}{c}19.00 \\
(4.36)^{\text {bcde }} \\
\end{array}$ & \begin{tabular}{|c|}
13.67 \\
$(3.69)$ bcde \\
\end{tabular} & $\begin{array}{c}18.67 \\
(4.23)^{\text {bcd }} \\
\end{array}$ \\
\hline $\mathrm{T}_{9}$ & $1.70 \mathrm{ml}$ & $\begin{array}{c}5.67 \\
(2.33) \\
\end{array}$ & $\begin{array}{c}0.33 \\
(0.88)^{\mathrm{d}} \\
\end{array}$ & $\begin{array}{c}0.33 \\
(0.88)^{\text {bc }} \\
\end{array}$ & $\begin{array}{c}5.00 \\
(2.12)^{\mathrm{bcd}} \\
\end{array}$ & $\begin{array}{c}1.67 \\
(1.38)^{\text {de }} \\
\end{array}$ & \begin{tabular}{|c|}
14.33 \\
$(3.73)^{\text {bcd }}$ \\
\end{tabular} & $\begin{array}{c}26.33 \\
(4.92)^{\mathrm{bcd}} \\
\end{array}$ & $\begin{array}{c}13.33 \\
(3.64)^{\mathrm{bc}} \\
\end{array}$ & $\begin{array}{c}14.00 \\
(3.62)^{\text {cde }}\end{array}$ & $\begin{array}{c}31.67 \\
(5.54)^{\mathrm{bcd}} \\
\end{array}$ & $\begin{array}{c}22.00 \\
(4.57)^{\mathrm{abc}} \\
\end{array}$ & $\begin{array}{l}14.00 \\
(3.73)^{\mathrm{f}}\end{array}$ & \begin{tabular}{|c|}
14.67 \\
$(5.54)^{\text {bcd }}$ \\
\end{tabular} & $\begin{array}{c}19.00 \\
(4.34)^{\text {bcde }} \\
\end{array}$ & $\begin{array}{c}16.67 \\
(4.06){ }^{\mathrm{bc}} \\
\end{array}$ & $\begin{array}{c}17.67 \\
(4.17)^{\mathrm{bcd}} \\
\end{array}$ \\
\hline $\mathrm{T}_{10}$ & $0.50 \mathrm{ml}$ & $\begin{array}{c}6.00 \\
(2.53) \\
\end{array}$ & $\begin{array}{c}1.67 \\
(1.35)^{\mathrm{bcd}} \\
\end{array}$ & $\begin{array}{c}0.33 \\
(0.88)^{\mathrm{bc}} \\
\end{array}$ & $\begin{array}{c}6.67 \\
(2.39) \mathrm{bc} \\
\end{array}$ & $\begin{array}{c}7.00 \\
(2.70)^{\mathrm{bc}} \\
\end{array}$ & \begin{tabular}{|c|}
10.67 \\
$(3.33)^{\text {bcd }}$ \\
\end{tabular} & \begin{tabular}{|c|}
31.67 \\
$(5.60)^{\mathrm{bc}}$ \\
\end{tabular} & $\begin{array}{c}13.00 \\
(3.55)^{\mathrm{bc}} \\
\end{array}$ & $\begin{array}{c}36.00 \\
(5.87)^{\mathrm{ab}} \\
\end{array}$ & \begin{tabular}{|c|}
41.00 \\
$(6.20)^{\text {bcd }}$ \\
\end{tabular} & $\begin{array}{c}19.67 \\
(4.34)^{\mathrm{bc}} \\
\end{array}$ & $\begin{array}{c}23.00 \\
(4.79)^{\text {cde }} \\
\end{array}$ & \begin{tabular}{|c|}
19.00 \\
$(6.20)^{\text {bcd }}$ \\
\end{tabular} & $\begin{array}{c}18.00 \\
(4.21)^{\text {cde }} \\
\end{array}$ & \begin{tabular}{|c|}
16.33 \\
$(3.96)^{\mathrm{bcd}}$ \\
\end{tabular} & $\begin{array}{c}23.00 \\
(4.76)^{\mathrm{b}} \\
\end{array}$ \\
\hline $\mathrm{T}_{11}$ & $1.0 \mathrm{~g}$ & $\begin{array}{l}6.00 \\
(2.3) \\
\end{array}$ & $\begin{array}{c}0.67 \\
(1.05)^{\mathrm{cd}}\end{array}$ & $\begin{array}{c}0.66 \\
(0.99)^{\mathrm{bc}} \\
\end{array}$ & $\begin{array}{c}6.67 \\
(2.64)^{\mathrm{b}} \\
\end{array}$ & $\begin{array}{c}5.50 \\
(2.04)^{\mathrm{bcd}} \\
\end{array}$ & \begin{tabular}{|c|}
12.33 \\
$(3.42)^{\mathrm{bcd}}$ \\
\end{tabular} & $\begin{array}{c}32.67 \\
(5.55)^{\mathrm{bc}} \\
\end{array}$ & $\begin{array}{c}14.00 \\
(3.60)^{\mathrm{bc}}\end{array}$ & $\begin{array}{c}25.67 \\
(4.70)^{\mathrm{bcd}} \\
\end{array}$ & $\begin{array}{c}38.00 \\
(6.16)^{\mathrm{bcd}}\end{array}$ & $\begin{array}{c}12.00 \\
(3.45)^{\mathrm{cd}} \\
\end{array}$ & $\begin{array}{c}19.33 \\
(4.39)^{\mathrm{e}} \\
\end{array}$ & $\begin{array}{c}19.00 \\
(6.16)^{\mathrm{bcd}}\end{array}$ & $\begin{array}{c}18.67 \\
(4.32)^{\text {bcde }} \\
\end{array}$ & $\begin{array}{c}10.67 \\
(3.22)^{\text {cde }} \\
\end{array}$ & $\begin{array}{c}21.00 \\
(4.55)^{\mathrm{bc}} \\
\end{array}$ \\
\hline $\mathrm{T}_{12}$ & Control & $\begin{array}{c}9.00 \\
(2.85) \\
\end{array}$ & $\begin{array}{c}8.33 \\
(2.97)^{\mathrm{a}} \\
\end{array}$ & $\begin{array}{c}7.33 \\
(2.80)^{\mathrm{a}} \\
\end{array}$ & $\begin{array}{c}16.67 \\
(4.12)^{\mathrm{a}} \\
\end{array}$ & $\begin{array}{c}21.00 \\
(3.76)^{a} \\
\end{array}$ & \begin{tabular}{|c|}
42.67 \\
$(6.28)^{\mathrm{a}}$ \\
\end{tabular} & \begin{tabular}{|c|}
63.67 \\
$(5.68)^{\mathrm{a}}$ \\
\end{tabular} & $\begin{array}{c}32.00 \\
(5.58)^{\mathrm{a}} \\
\end{array}$ & $\begin{array}{c}52.33 \\
(6.79)^{\mathrm{a}} \\
\end{array}$ & \begin{tabular}{|c|}
77.00 \\
$(8.58)^{\mathrm{a}}$ \\
\end{tabular} & $\begin{array}{c}34.33 \\
(5.86)^{\mathrm{a}} \\
\end{array}$ & $\begin{array}{c}40.00 \\
(6.31)^{\mathrm{a}} \\
\end{array}$ & \begin{tabular}{|c|}
37.67 \\
$(8.62)^{\mathrm{a}}$ \\
\end{tabular} & $\begin{array}{c}35.67 \\
(5.95)^{\mathrm{a}} \\
\end{array}$ & \begin{tabular}{|c|}
32.67 \\
$(5.72)^{\mathrm{a}}$ \\
\end{tabular} & $\begin{array}{c}34.67 \\
(5.88)^{\mathrm{a}} \\
\end{array}$ \\
\hline S.Em \pm & & & 0.22 & 0.23 & 0.47 & 0.34 & 0.77 & 0.64 & 0.51 & 0.66 & 0.65 & 0.46 & 0.20 & 0.65 & 0.31 & 0.30 & 0.26 \\
\hline $\mathrm{CD}(\mathrm{p}=0.05)$ & & NS & 0.62 & 0.68 & 1.38 & 0.98 & 2.26 & 1.88 & 1.47 & 1.94 & 1.89 & 1.34 & 0.60 & 1.91 & 0.90 & 0.90 & 0.77 \\
\hline
\end{tabular}

$\mathrm{T}_{1}$ : Azadiracthin $1500 \mathrm{ppm}, \mathrm{T}_{2}$ : Lecanicillium lecanii, $\mathrm{T}_{3}$ : Imidacloprid 45 FS (Seed Treatment) + Acephate 75 SP (Foliar spray), $\mathrm{T}_{4}$ : Diafenthiuron 50 WP, $\mathrm{T}_{5}$ :

Fipronil 5 SC

$\mathrm{T}_{6}$ : Abamectin 1.9 SC, $\mathrm{T}_{7}$ : Imidacloprid $30.50 \mathrm{SC}, \mathrm{T}_{8}$ : Thiamethoxam $25 \mathrm{WG}, \mathrm{T} 9$ : Dimethoate 30 EC, $\mathrm{T}_{10}$ : Lambda cyahalothrin 5 EC, T11: Acephate 75 SP,

$\mathrm{T}_{12}$ : Untreated control

* Mean of 3 Replications Figures in parenthesis indicate square root $\sqrt{\mathrm{x}+1}$ transformed values Figures in each column followed by same alphabet (s) are not significantly different $(\mathrm{P}=0.05)$

NS- Non significant 
Table.3 Bio-efficacy of different synthetic insecticides, bio-pesticide and botanical pesticide against Thrips palmi on watermelon crop (Pooled mean)

\begin{tabular}{|c|c|c|c|}
\hline Treatments & $\begin{array}{c}\text { Pooled } \\
\text { mean }\end{array}$ & Sprays & $\begin{array}{c}\text { Pooled } \\
\text { mean }\end{array}$ \\
\hline $\begin{array}{l}\text { Azadiracthin } 1500 \text { ppm } \\
\text { Lecanicillium (=Verticillium) lecanii }\end{array}$ & $\begin{array}{c}21.62 \\
(4.30)^{\mathrm{de}} \\
22.91 \\
(4.45)^{\mathrm{e}}\end{array}$ & I & $\begin{array}{c}2.68 \\
(1.51)^{\mathrm{a}}\end{array}$ \\
\hline $\begin{array}{l}\text { Imidacloprid } 45 \text { FS (Seed Treatment)+ Acephate } \\
75 \text { SP (Foliar spray) } \\
\text { Diafenthiuron } 50 \mathrm{WP}\end{array}$ & $\begin{array}{c}6.29 \\
(2.26)^{\mathrm{a}} \\
10.78 \\
(3.01)^{\mathrm{b}}\end{array}$ & II & $\begin{array}{c}17.52 \\
(3.67)^{b}\end{array}$ \\
\hline $\begin{array}{l}\text { Fipronil } 5 \mathrm{SC} \\
\text { Abamectin } 1.9 \mathrm{SC}\end{array}$ & $\begin{array}{c}11.40 \\
(3.07)^{\mathrm{b}} \\
15.62 \\
(3.65)^{\mathrm{c}}\end{array}$ & III & $\begin{array}{c}25.63 \\
(4.67)^{d}\end{array}$ \\
\hline $\begin{array}{l}\text { Imidacloprid } 30.50 \mathrm{SC} \\
\text { Thiamethoxam } 25 \mathrm{WG}\end{array}$ & $\begin{array}{c}11.04 \\
(3.09)^{\mathrm{b}} \\
17.91 \\
(3.76)^{\mathrm{c}}\end{array}$ & IV & $\begin{array}{c}19.89 \\
(4.40)^{d}\end{array}$ \\
\hline $\begin{array}{l}\text { Dimethoate } 30 \mathrm{EC} \\
\text { Lambda cyhalothrin } 5 \mathrm{EC}\end{array}$ & $\begin{array}{c}14.06 \\
(3.44)^{\mathrm{bc}} \\
17.80 \\
(3.92)^{\mathrm{cd}}\end{array}$ & V & $\begin{array}{c}18.02 \\
(4.19)^{c}\end{array}$ \\
\hline $\begin{array}{l}\text { Acephate } 75 \text { SP (Standard check) } \\
\text { Untreated control }\end{array}$ & $\begin{array}{c}15.79 \\
(3.65)^{\mathrm{c}} \\
35.73 \\
(5.67)^{\mathrm{f}}\end{array}$ & & \\
\hline
\end{tabular}

\begin{tabular}{|l|c|c|}
\hline \multicolumn{1}{|c|}{ Factors } & SEm \pm & CD at 5\% \\
\hline Spray & 0.108 & 0.304 \\
Treatments & 0.327 & 0.915 \\
Spray X Treatments & 1.306 & 3.658 \\
\hline
\end{tabular}

* Mean of 3 Replications Figures in parenthesis indicate square root $(\sqrt{\mathrm{x}+1})$ transformed values Figures in each column followed by same alphabet $(\mathrm{s})$ are not significantly different $(\mathrm{P}=0.05)$ 
Table.4 Effect of synthetic insecticides, bio-pesticide and botanical pesticide on WBNV disease incidence in watermelon

\begin{tabular}{|c|c|c|c|c|c|c|c|}
\hline \multirow{2}{*}{ Treatments } & \multirow{2}{*}{ Before Spray } & \multicolumn{5}{|c|}{ *Mean Per cent WBNV disease incidence } & \multirow{2}{*}{ Pooled mean } \\
\hline & & I Spray & II Spray & III Spray & IV Spray & V Spray & \\
\hline Azadiracthin 1500 ppm & $\begin{array}{c}8.27 \\
(16.25) \\
\end{array}$ & $\begin{array}{c}20.95 \\
(26.56) \\
\end{array}$ & $\begin{array}{c}40.33 \\
(39.22) \\
\end{array}$ & $\begin{array}{c}81.11 \\
(64.24) \\
\end{array}$ & $\begin{array}{l}100.00 \\
(90.00) \\
\end{array}$ & $\begin{array}{l}100.00 \\
(90.00) \\
\end{array}$ & $\begin{array}{c}68.48 \\
(62.00)\end{array}$ \\
\hline Lecanicillium lecanii & $\begin{array}{c}5.95 \\
(13.80) \\
\end{array}$ & $\begin{array}{c}27.25 \\
(31.43) \\
\end{array}$ & $\begin{array}{c}42.01 \\
(40.39) \\
\end{array}$ & $\begin{array}{c}77.60 \\
(61.83) \\
\end{array}$ & $\begin{array}{r}100.00 \\
(90.00) \\
\end{array}$ & $\begin{array}{r}100.00 \\
(90.00) \\
\end{array}$ & $\begin{array}{c}69.37 \\
(62.73)^{\mathrm{cd}}\end{array}$ \\
\hline $\begin{array}{l}\text { Imidacloprid } 45 \text { FS (Seed Treatment) + Acephate } 75 \text { SP (Foliar } \\
\text { spray) }\end{array}$ & $\begin{array}{c}8.06 \\
(15.19) \\
\end{array}$ & $\begin{array}{c}5.81 \\
(13.64) \\
\end{array}$ & $\begin{array}{c}21.42 \\
(27.54) \\
\end{array}$ & $\begin{array}{c}42.19 \\
(40.50) \\
\end{array}$ & $\begin{array}{c}95.25 \\
(77.90) \\
\end{array}$ & $\begin{array}{c}98.89 \\
(86.50) \\
\end{array}$ & $\begin{array}{c}52.71 \\
(49.21)^{\mathrm{a}} \\
\end{array}$ \\
\hline Diafenthiuron $50 \mathrm{WP}$ & $\begin{array}{c}6.67 \\
(14.68) \\
\end{array}$ & $\begin{array}{c}11.11 \\
(19.42) \\
\end{array}$ & $\begin{array}{c}17.69 \\
(24.85) \\
\end{array}$ & $\begin{array}{c}67.22 \\
(55.10) \\
\end{array}$ & $\begin{array}{c}98.33 \\
(84.02) \\
\end{array}$ & $\begin{array}{c}99.44 \\
(87.52) \\
\end{array}$ & $\begin{array}{c}58.76 \\
(54.18)\end{array}$ \\
\hline Fipronil $5 \mathrm{SC}$ & $\begin{array}{c}4.87 \\
(12.46) \\
\end{array}$ & $\begin{array}{c}21.18 \\
(27.38) \\
\end{array}$ & $\begin{array}{c}35.21 \\
(36.28) \\
\end{array}$ & $\begin{array}{c}70.58 \\
(57.18) \\
\end{array}$ & $\begin{array}{r}100.00 \\
(90.00) \\
\end{array}$ & $\begin{array}{r}100.00 \\
(90.00) \\
\end{array}$ & $\begin{array}{c}65.40 \\
(60.16)\end{array}$ \\
\hline Abamectin 1.9 SC & $\begin{array}{c}6.59 \\
(14.64) \\
\end{array}$ & $\begin{array}{c}30.88 \\
(33.53) \\
\end{array}$ & $\begin{array}{c}38.70 \\
(38.39) \\
\end{array}$ & $\begin{array}{c}76.43 \\
(61.51) \\
\end{array}$ & $\begin{array}{l}100.00 \\
(90.00) \\
\end{array}$ & $\begin{array}{l}100.00 \\
(90.00) \\
\end{array}$ & $\begin{array}{c}69.20 \\
(62.69)^{\mathrm{cd}}\end{array}$ \\
\hline Imidacloprid $30.50 \mathrm{SC}$ & $\begin{array}{c}6.60 \\
(14.88) \\
\end{array}$ & $\begin{array}{c}9.82 \\
(17.79) \\
\end{array}$ & $\begin{array}{c}28.05 \\
(31.95) \\
\end{array}$ & $\begin{array}{c}62.09 \\
(52.02) \\
\end{array}$ & $\begin{array}{c}97.81 \\
(81.59) \\
\end{array}$ & $\begin{array}{c}99.44 \\
(87.52) \\
\end{array}$ & $\begin{array}{c}59.44 \\
(54.17)\end{array}$ \\
\hline Thiamethoxam $25 \mathrm{WG}$ & $\begin{array}{c}7.35 \\
(15.52) \\
\end{array}$ & $\begin{array}{c}26.53 \\
(30.98) \\
\end{array}$ & $\begin{array}{c}43.11 \\
(41.03) \\
\end{array}$ & $\begin{array}{c}70.75 \\
(57.35) \\
\end{array}$ & $\begin{array}{c}100.00 \\
(90.00)\end{array}$ & $\begin{array}{l}100.00 \\
(90.00)\end{array}$ & $\begin{array}{c}68.10 \\
(61.87)^{\mathrm{c}}\end{array}$ \\
\hline Dimethoate $30 \mathrm{EC}$ & $\begin{array}{c}6.38 \\
(14.56) \\
\end{array}$ & $\begin{array}{c}25.37 \\
(29.97) \\
\end{array}$ & $\begin{array}{c}43.39 \\
(40.97) \\
\end{array}$ & $\begin{array}{c}69.60 \\
(56.59) \\
\end{array}$ & $\begin{array}{r}100.00 \\
(90.00) \\
\end{array}$ & $\begin{array}{l}100.00 \\
(90.00) \\
\end{array}$ & $\begin{array}{c}67.67 \\
(61.51)^{c}\end{array}$ \\
\hline Lambda cyhalothrin $5 \mathrm{EC}$ & $\begin{array}{c}8.16 \\
(16.60)\end{array}$ & $\begin{array}{c}32.92 \\
(34.87) \\
\end{array}$ & $\begin{array}{c}44.45 \\
(41.80)\end{array}$ & $\begin{array}{c}76.22 \\
(61.06)\end{array}$ & $\begin{array}{c}98.94 \\
(86.58)\end{array}$ & $\begin{array}{l}100.00 \\
(90.00)\end{array}$ & $\begin{array}{c}70.50 \\
(62.86)^{\mathrm{cd}}\end{array}$ \\
\hline Acephate 75 SP (Standard check) & $\begin{array}{c}8.16 \\
(16.55) \\
\end{array}$ & $\begin{array}{c}17.37 \\
(24.62) \\
\end{array}$ & $\begin{array}{c}37.48 \\
(37.74) \\
\end{array}$ & $\begin{array}{c}78.25 \\
(62.25) \\
\end{array}$ & $\begin{array}{c}98.90 \\
(85.09) \\
\end{array}$ & $\begin{array}{l}100.00 \\
(90.00) \\
\end{array}$ & $\begin{array}{c}66.40 \\
(59.94)\end{array}$ \\
\hline Untreated control & $\begin{array}{c}7.60 \\
(15.99) \\
\end{array}$ & $\begin{array}{c}35.92 \\
(36.80) \\
\end{array}$ & $\begin{array}{c}46.91 \\
(43.20)\end{array}$ & $\begin{array}{c}84.79 \\
(66.98) \\
\end{array}$ & $\begin{array}{c}100.00 \\
(90.00) \\
\end{array}$ & $\begin{array}{c}100.00 \\
(90.00) \\
\end{array}$ & $\begin{array}{c}73.51 \\
(65.39)\end{array}$ \\
\hline Pooled mean & - & $\begin{array}{c}22.09 \\
(27.25)^{a}\end{array}$ & $\begin{array}{c}36.56 \\
(36.94)\end{array}$ & $\begin{array}{c}71.40 \\
(58.05)\end{array}{ }^{\mathrm{c}}$ & $\begin{array}{c}99.10 \\
(87.09)\end{array}$ & $\begin{array}{c}99.81 \\
(89.29)\end{array}$ & \\
\hline
\end{tabular}

\begin{tabular}{|c|c|c|}
\hline Factors & SEm \pm & CD at 5\% \\
\hline Spray & 0.108 & 0.304 \\
Treatments & 0.327 & 0.915 \\
Spray X Treatments & 1.306 & 3.658 \\
\hline
\end{tabular}

* Mean of 3 Replications; Figures in parenthesis indicate Arc sine transformed values

Treatment pooled mean in the column followed by same alphabet (s) are not significantly different $(\mathrm{P}=0.05)$ by DMRT

Spray pooled mean in each row followed by same alphabet $(s)$ are not significantly different $(\mathrm{P}=0.05)$ by DMRT 
Similar trend was also noticed with WBNV disease incidence. The Imidacloprid @ $10 \mathrm{ml}$ per $\mathrm{kg}$ seeds as seed treatment followed by foliar application of Acephate @ $1.00 \mathrm{~g} / \mathrm{l}$ of water could manage the disease incidence effectively up to about 60 days after sowing and it was statistically significant from all other treatments, followed by Diafenthiuron @ $1.25 \mathrm{~g} / \mathrm{l}$ of water and Imidacloprid @ 0.30 $\mathrm{ml} / \mathrm{l}$ water as foliar sprays (Table 4).

All the treatments were on par with each other at seventh day after fifth spray. The results indicated that chemical control of thrips may not be an effective answer for management of WBNV disease incidence.

Similar trend was noticed with respect to the performance of various treatments on WBNV disease incidence when the data were analysed using two factorial analyses. Among the different treatments the lowest pooled mean per cent disease incidence of 52.71 was recorded in plants treated with imidacloprid as seed treatment followed by foliar application of acephate, whereas highest pooled mean per cent disease incidence of 69.37 was recorded with plants treated with a bio-pesticide, Lecanicillium lecanii (Table 4).

A field experiment conducted during summer season of 2014 revealed that, as the crop stage advanced the extent of thrips infestation and WBNV disease incidence also increased irrespective of the treatments. Thereafter, the thrips population declined gradually. The overall disease incidence in the plots assigned to different treatments did not exceed 45 per cent including control plots at initial stage of the crop growth i.e. up to 60 days after sowing. Thereafter, the disease incidence increased significantly and reached about 100 per cent in all the treatment plots within a short period of time.

Krupashankar (1998) also observed similar results during the survey carried out in different districts of Karanataka mainly Bangaluru, Kolar, Chitradurga and Mandya. The disease incidence ranged between 0.7 to 10 per cent up to 30-45 days after sowing and 10 to100 per cent up to 60-100 days after sowing. Similar observations were also noticed by Krishnakumar et al., (2006) from watermelon experimental fields at IIHR, who reported that, the WBNV infection was not visual up to three weeks after sowing of watermelon, irrespective of the month of sowing. But by the fourth week, the infection rose up to 4 to 16 per cent in all plantings and later it spread to nearly 100 per cent by eleventh week. The Imidacloprid @ $10 \mathrm{ml}$ per $\mathrm{kg}$ seeds as seed treatment followed by foliar spraying of Acephate @ $1.00 \mathrm{~g} / \mathrm{l}$ of water was found to be superior in reducing the thrips population throughout the cropping period. Next best treatments in the order were Diafenthiuron $1.25 \mathrm{~g} / \mathrm{l}$ of water and Imidacloprid $0.30 \mathrm{ml} / 1$ of water as foliar sprays.

Similar trend was also noticed with WBNV disease incidence. The Imidacloprid @ $10 \mathrm{ml}$ per $\mathrm{kg}$ seeds as seed treatment followed by foliar application of Acephate @ $1.00 \mathrm{~g} / \mathrm{l}$ of water could manage the disease incidence effectively up to about 60 days after sowing and it was statistically significant from all other treatments, followed by Diafenthiuron @ $1.25 \mathrm{~g} / \mathrm{l}$ of water and Imidacloprid @ 0.30 $\mathrm{ml} / \mathrm{l}$ water as foliar sprays.

Results of the present study are in agreement with the findings of Rajasekharam (2010) where the treatments involving seed treatment with Imidacloprid, planting of maize in between, use of silver colour UV reflective mulch along with alternate chemical sprays of Imidacloprid and Thiomethoxam on crop and/or on weed hosts on bunds were found to be more effective in reducing the vector population and thus minimizing the secondary spread of WBNV on water melon. It is thus inferred that not only the control of vector but 
also the destruction of weed hosts or spraying insecticides is a useful and effective strategy in the integrated management of watermelon bud necrosis disease.

Similar findings were also obtained by Krishnakumar et al., (2006) during a field experiment conducted at Indian Institute of Horticultural Research, Bangalore, to manage the thrips on watermelon by using a combination of synthetic and botanical insecticides. Results revealed that covering soil with black polythene mulch, seed treatment with Imidacloprid (Gaucho $75 \mathrm{WS}$ $3 \mathrm{gm} / \mathrm{kg}$ of seed) and Acephate $500 \mathrm{~g}$ a.i./ha spray at 10 days interval significantly reduced thrips infestation. Results of the present study were also in the line of work carried out during 2006 and 2007 by Kamanna et al., (2010). Among the different insecticides tested alone and in combinations, the seed treatment with Imidacloprid $70 \mathrm{WS}$ at $10 \mathrm{~g} / \mathrm{kg}$ of seeds + Oxydemeton-methyl $25 \mathrm{EC}$ at 1.5 $\mathrm{ml} /$ lit spray recorded lowest incidence of WBNV (19.67\%) and thrips infestation as well as highest yield (70.44 t/ha) as compared to other insecticides and untreated control. Authors strongly opined that seed treatment or spraying of insecticides alone was not effective. An integrated approach of seed treatment with Imidacloprid followed by spraying of systemic insecticides for the control of thrips is the most effective method.

It was observed in the present field experiment that the management of thrips and WBNV disease by application of botanicals and a fungal based pesticide Lecanicillium lecanii was relatively less effective that of other synthetic insecticides. It can be attributed to the reason that these products could not reduce the population of vectors effectively and thus failing to reduce the secondary spread as the crop stage advanced. Similar opinion was also expressed by Krishnakumar et al., (2006) and Rajasekharam (2010) that application of botanical extracts and bio-pesticdes were found to be least effective in managing thrips as well as WBNV disease as compared to the other integrated management approaches.

Among the different insecticide molecules tested seed treatment with imidacloprid, along with foliar sprays of acephate were found to be most effective in managing the thrips and WBNV on watermelon crop by minimizing the vector population, thus checking secondary spread of disease on the crop.

Next best synthetic chemicals in the order of effectiveness were diafenthiuron, imidacloprid, thiomethoxam and abamectin as foliar sprays. It was observed in the present study that the application of azadirachtin and a fungal based bio-pesticide, Lecanicillium lecanii was relatively less effective in managing thrips and WBNV disease as compared to other synthetic insecticides.

\section{References}

Bangalore (India), p. 76.

FAOSTAT, FAO Production statistics. http://www.faostat.fao.org, Accessed on March, 2014.

Kamanna, B.C., Jadhav, S.N. and Shankarappa, T.H., 2010. Evaluation of insecticides against thrips vector for the management of watermelon bud necrosis virus (WBNV) disease. Karnataka Journal of Agriculture Science, 23: 172-173.

Krishnakumar, N.K., Venkatesh, N., Kalleshwaraswamy, C.M. and Ranganath, H.R., 2006. Seasonal incidence of thrips and bud necrosis virus on water melon. Pest Management in Horticultural Eco-Systems, 12: 8592.

Krupashankar, M. R., 1998. Studies on bud necrosis virus disease of watermelon (Citrullus lanatus Thunb.). M. Sc. (Ag.) Thesis, University of Agricultural 
Sciences,

Mound, L. A., 1996. The Thysanoptera Vector Species of Tospoviruses. Acta Horticulture, 431: 298- 309.

Rajasekharam, T., 2010. Biological and molecular characterization and management of watermelon bud necrosis virus. Ph.D. Thesis, University of Agricultural Sciences, Dharwad,
Karnataka. p: 142.

Singh, S.J., and Krishnareddy, M., 1995. Thrips flavus Schrank (Thysanoptera: Thripidae), a new insect vector of a tospo virus infecting watermelon in India. Pest Management in Horticultural Ecosystems, 1(2): 115118.

\section{How to cite this article:}

Shruthi, C.R., G.B. Narabenchi, B.S. Rohini and Chethan Nandi. 2017. Bioefficacy of Synthetic Insecticides, Bio-Pesticide and Botanical against Thrips and Watermelon Bud Necrosis Viral Disease on Watermelon. Int.J.Curr.Microbiol.App.Sci. 6(9): 540-548. doi: https://doi.org/10.20546/ijcmas.2017.609.065 